\title{
Utilização de Técnicas de Aprendizado de Máquina para Acompanhamento de Fóruns Educacionais
}

\author{
Title: Monitoring Educational Forums using Machine Learning
}

Vitor Belarmino Rolim

Universidade Federal Rural de

Pernambuco

victor.b.rolim@gmail.com

\author{
Rafael Ferreira Leite de Mello \\ Universidade Federal Rural de \\ Pernambuco \\ rafaelflmello@gmail.com
}

\author{
Evandro de Barros Costa \\ Universidade Federal de \\ Alagoas \\ ebc.academico@gmail.com
}

\section{Resumo}

Com o crescimento da educação à distância, e o uso da tecnologia como ferramenta de apoio educacional, a utilização dos ambientes virtuais de aprendizagem também cresceu. Esses ambientes oferecem vários recursos que podem ser utilizados para auxiliar no processo ensino-aprendizagem, sendo o fórum um desses recursos. No entanto, muitas vezes o uso de fóruns de discussão em plataformas educacionais online requer a necessidade de acompanhamento de centenas ou até milhares de usuários. Devido à grande quantidade de postagens, é dificil para o professor ou tutor acompanhar os alunos. Para tentar contornar esse problema, buscando um acompanhamento eficiente, procura-se disponibilizar ferramentas computacionais que auxiliem o professor. Este trabalho tem como objetivo auxiliar professores e alunos na participação de fóruns educacionais através de classificação de postagem e recomendação de conteúdo. Para tanto, foram consideradas duas etapas: identificação de postagens de dúvida e extração do assunto da postagem, as quais foram avaliadas em um estudo experimental. Na etapa de identificação, conseguiu-se um percentual de acerto na classificação de 97\%, fazendose uso de um algoritmo baseado na rede neural. Na etapa de extração, foi implementado um algoritmo baseado em aprendizagem não supervisionada e no Wikipédia. Este algoritmo alcançou uma acurácia de 76,1\%. Com base no assunto extraído da postagem, o sistema recomenda vídeo para o aluno. Concluiu-se, portanto, que essa abordagem auxilia tanto o professor, reduzindo o tempo empregado para responder todos os questionamentos dos alunos, quanto o aluno, fornecendo materiais de estudo para auxiliar a resolver o seu questionamento.

Palavras-Chave: Ambientes Virtuais de Aprendizagem, classificação de texto, extração de assunto, sistema de recomendação.

\begin{abstract}
With the growth of distance education, and the adoption of computational systems as an educational support tool, the use of virtual learning environments has also grown. These environments offer various tools that could be used to assist in the teaching-learning process, one of these tools is the forum. Discussion forums in online educational platforms often require monitoring of thousands of users. Due to the large number of posts, it is difficult to supervise all students. In order to perform an effective monitoring, it is necessary computational tools to assist the teacher. This work aims to develop an approach to aid teachers and students to manage educational forums through post classification and content recommendation. To achieve this goal, two steps were performed: the identification of posts classified as question, and the extraction of the subject from post. In the Identification stage, the neural network algorithm achieved 97\% of accuracy. For subject extraction step was proposed an algorithm based on unsupervised learning and Wikipedia. This algorithm achieved a 76.1\% of accuracy. Moreover, the system recommended videos to the student according to the subject extracted from posts. This approach assists the teacher by reducing the time used to answer the students' questions, and the student by providing supplementary materials to help solve his question.
\end{abstract}

Keywords: Virtual Learning Environments, text classification, subject extraction, recommendation system.

Cite as: Rolim, V., Ferreira, R. \& Costa, E. (2017). Monitoring Educational Forums using Machine Learning (Utilização de Técnicas de Aprendizado de Máquina para Acompanhamento de Fóruns Educacionais). Brazilian Journal of Computers in Education (Revista Brasileira de Informática na Educação - RBIE), 25(3), 112-130. DOI: 10.5753/RBIE.2017.25.03.112 


\section{Introdução}

Devido à popularização do uso da tecnologia aplicada a educação, houve também a difusão dos ambientes virtuais de aprendizagem (AVA) [Jain 2015]. Os AVAs possuem várias ferramentas que auxiliam alunos e professores no desenvolvimento do conhecimento, na interação e na avaliação do desempenho. Alguns exemplos dessas ferramentas são: wikis, fóruns, blogs, redes sociais, etc. Tais ferramentas, potencialmente, possuem um importante papel na atratividade e no aumento de interação na disciplina trabalhada pelo professor. Assim, é importante que os AVAs ofereçam suporte ao uso efetivo dessas ferramentas, contribuindo na interação entre aluno e tutor/professor, particularmente provendo meios de acompanhamento direto e indireto para garantir o aprendizado do aluno [Akyuz e Kurt 2010].

O acompanhamento direto é aquele realizado sob a supervisão do professor ou tutor. Para isso, cada curso tem, normalmente, um plano de ensino e cronograma de atividades que são acompanhados de perto pelos professores e tutores. Então, todo material e discussões disponibilizados no AVA são verificados manualmente para que as dúvidas dos alunos sejam resolvidas e seus progressos computados. Devido à grande quantidade de dados, esse tipo de acompanhamento se torna, por vezes, inviável de ser realizado.

Por outro lado, o acompanhamento indireto é realizado sem a participação ativa do professor. Ele é uma alternativa para a problemática da sobrecarga de informações geradas nos AVAs. Para isso, é importante ter sistemas automatizados que possam auxiliar o professor nessa atividade [Rigo et al. 2013].

Em particular, a ferramenta assíncrona de fórum tem sido bastante utilizada por permitir uma grande interatividade entre alunos e professores [Barros e Carvalho 2011], podendo ser usada para postar: dúvidas, comentários sobre a disciplina, outras fontes de assunto, possíveis respostas para questões levantadas pelo professor, entre outros. Cheng et al. (2011) realizaram um estudo que mostra a efetividade da ferramenta de fórum para melhorar o desempenho de estudantes em um AVA. Contudo, com o aumento da interação entre os usuários do fórum, ocorre um aumento também do volume das postagens, tornando-se por vezes inviável o gerenciamento de todas elas pelo professor, constituindo-se, assim, um problema de pesquisa relevante e desafiador. Para lidar com esse problema, busca-se desenvolver ferramentas computacionais para analisar automaticamente postagens de fóruns educacionais [Azevedo et al. 2011a].

$\mathrm{Na}$ perspectiva do problema destacado, a literatura em Informática na Educação apresenta vários trabalhos que extraem informações específicas de fóruns, como: Classificação de postagens [Rolim et al. 2016a, Azevedo et al. 2011a, Gerosa et. al. 2003], sistema de recomendação de materiais para alunos aluno [Baker e Yacef 2009, Mohamad e Tasir 2013], análise das discussões dos alunos [Pena-Shaff e Nicholls 2004], avaliação de desempenho de estudantes [Romero et al. 2013, Yoo e Kim 2014], entre outros. Porém, diante da grande variedade de aplicações educacionais dos fóruns, ainda se faz necessária a criação de sistemas computacionais para lidar com a quantidade de dados gerados.

Neste trabalho, propõe-se uma abordagem com um sistema automático para auxiliar o professor na resposta a dúvidas de alunos [Rolim et al. 2016b]. Para isso, o sistema realiza:

1. A identificação do tipo de postagem feita no fórum educacional. Foi proposta uma abordagem baseada em aprendizagem de máquina para classificar as postagens em resposta, comentário neutro e dúvida. 
2. A extração do assunto contido na postagem. Para essa etapa foi proposto um sistema não supervisionado baseado na ementa inicial da disciplina e em páginas da Wikipédia.

Ademais, caso o sistema identifique postagens de dúvidas com assuntos relacionados à disciplina, é realizada a recomendação de um material de estudo auxiliar para o aluno. Além disto, o professor tem acesso a estatísticas sobre quais os principais assuntos que a turma está com dificuldade. Para tal foram feitos testes em três diferentes bancos de dados de fóruns educacionais, contendo no total 1487 postagens.

O resto do artigo é apresentado como segue: A Seção 2 apresenta um breve embasamento sobre aprendizagem de máquina, recuperação de informação e técnicas de préprocessamento textuais utilizadas no trabalho. Os trabalhos relacionados são abordados na Seção 3. A Seção 4 contém a proposta desse trabalho e como ela foi executada. Na Seção 5 são apresentados os resultados para as etapas do sistema. Por fim, na Seção 6 são ponderadas considerações finais e trabalhos futuros.

\section{Embasamento Teórico}

Esta seção apresenta os principais conceitos e discussões sobre técnicas relacionadas ao trabalho aqui proposto.

\subsection{Fóruns Educacionais}

O fórum de discussão é uma ferramenta de comunicação assíncrona online, tendo como função principal promover discussões entre os participantes acerca de diferentes temas. Essa ferramenta é frequentemente utilizada nos ambientes virtuais de aprendizagem, por ser um recurso que proporciona a interação coletiva dos participantes [De Wever et al. 2006]. Quando utilizado no meio educacional, fornece aos alunos um canal de comunicação, prestando-se para expressar suas dúvidas, opiniões e respostas aos questionamentos existentes sobre algum assunto. Por isso, comumente o fórum educacional possui professores ou tutores, como mediadores das interações, para que se possa auxiliar os alunos no processo de aprendizagem [Batista e Gobara 2007].

Um aspecto importante é que o fórum é considerado uma das ferramentas que permite maior interatividade entre alunos e professores. Segundo o estudo realizado por Barros e Carvalho (2011) o fórum foi apontado por $69,2 \%$ dos alunos como a ferramenta mais interativa. Outras ferramentas que também tem essa característica são: tarefa $(41,0 \%)$, chat $(38,5 \%)$ e questionário $(20,5 \%)$. 2009]:

Além disto, o professor pode usar o fórum em diversos contextos, por exemplo [Freitas

(i) incentivar a criação de laços entres os alunos a partir da discussão de temas específicos da disciplina;

(ii) desenvolver a capacidade de debate crítico acerca de algum tema ou assunto;

(iii) dar uma resposta sobre dúvidas e comentários;

(iv) guiar os estudos dos alunos baseados nas suas postagens;

(v) avaliar o aluno. 
O ganho pedagógico causado pela utilização dos fóruns educacionais é perceptível, mesmo que os alunos envolvidos no processo empreguem pouco tempo para utilizar esta ferramenta [Cheng et al. 2011, Mazzolini e Maddison 2007]. Contudo, como já foi mencionado, o aumento da interação entre os usuários do fórum faz crescer a quantidade de dados gerados pela ferramenta, o que dificulta o acompanhamento. Por isso, faz-se necessária a implementação de ferramentas que possam auxiliar o professor no processo de aprendizado dos alunos nos fóruns.

\subsection{Aprendizagem de Máquina}

Aprendizagem de máquina é uma das áreas da inteligência artificial, podendo ser definida como sistemas capazes de adquirir conhecimento a partir de dados. Weiss e Kulikowski (1991) definem um sistema de aprendizado de máquina como um programa de computador que toma decisões baseadas na experiência contida em exemplos anteriores.

Ela pode ser dividida em: (i) aprendizado supervisionado, o qual tem como objetivo compreender a relação entre as entradas e saídas fornecidas, para classificar, ou rotular, uma determinada instância (imagem, documento, etc.) em um conjunto de categorias pré-definidas; (ii) aprendizado não supervisionado, que tem como objetivo agrupar elementos com características similares. Entre suas diferentes aplicações, uma delas é a classificação textual.

A classificação textual tem como objetivo determinar se um documento específico pertence a uma ou mais classes [Sebastiani 2002]. Este processo é chamado de classificação ou categorização.

Classificação textual pode ser considerada uma abordagem que consiste em separar previamente uma coleção de documentos, identificando-os como pertencentes a uma classe específica. Posteriormente, um algoritmo de classificação, é treinado com essa coleção como dado de entrada. A partir deste treinamento o classificador é capaz de indicar a classe de uma entrada fornecida como teste.

Pode-se citar várias técnicas de aprendizagem de máquina com propósitos de classificação, tais como: Naive Bayes, Redes Neurais, Indução de Árvore de Decisão, entre outras. A seguir estão descritas as técnicas de classificação utilizadas no presente trabalho.

Naive Bayes é uma técnica probabilística baseada no Teorema de Bayes [Rutten 2010], sendo bastante utilizada em aprendizagem de máquina e reconhecimento de padrões. Esse classificador calcula a probabilidade de que uma amostra desconhecida pertença a cada uma das classes possíveis, predizendo a classe mais provável. Para isto, o classificador bayesiano calcula uma distribuição geradora para cada classe do problema através da análise das relações entre as características envolvidas e as classes de cada instância. Após treinar um classificador Bayesiano ele seleciona a classe com maior probabilidade.

Árvore de decisão [Quinlan 2014] é uma técnica indutiva de aprendizado de máquina que utiliza uma estrutura de árvore para avaliar os atributos de uma entrada e retorna uma predição baseada nos valores desses atributos. Essa árvore é estruturada através de vários nós, onde cada nó corresponde a um teste do valor de uma característica do dado de entrada. Os nós da árvore são ligados por ramos, os quais identificam os valores do teste realizado em cada nó. Por fim, cada nó da folha da árvore representa um valor de retorno. Sendo assim, a árvore de decisão chega a uma decisão através da realização de vários testes. Para isso, o algoritmo é iniciado na raiz da árvore e a percorre realizando testes sobre as características, que correspondem aos nós, do dado de entrada até chegar na folha da árvore. Ao chegar na folha da árvore é retornado como resultado a classificação.

A rede neural [Haykin 1999] é um modelo computacional inspirado nas ligações entre neurônios do cérebro humano. Esse modelo é composto por um conjunto de neurônios, ou nós, 
que são interligados entre si, formando uma rede. Esses neurônios são divididos em camadas e são conectados por meio de ligações. Cada ligação possui um peso, os nós são ajustados baseados nas características dos dados de entrada. A partir dos dados de entrada e da força de ligação entre os nós, a rede realiza a classificação dos dados entrada.

\subsection{Recuperação de Informação}

Recuperação de informação (RI) é uma subárea da ciência da computação que tem por objetivo recuperar dados, informações ou até mesmo documentos de uma determinada coleção de dados. Baeza-Yates e Ribeiro-Neto (2013) definem o objetivo principal de um sistema de RI como sendo a recuperação de todos os documentos que são relevantes à necessidade de informação do usuário. Grande parte da dificuldade encontrada nos sistemas de RI está em saber como extrair a informação dos documentos e verificar a relevância daquela informação.

\subsubsection{TF-IDF}

É a combinação de duas técnicas estatísticas, o TF (Term Frequency) e IDF (Inverse Document Frequency). Essa medida é frequentemente utilizada na mineração de texto.

$\mathrm{O}$ TF mede o quão frequente um termo ocorre em um documento. Pode ser representado pela divisão entre a quantidade de vezes que um termo aparece no documento (frequenciaT) e o total de termos que o documento possui $(\mathrm{N})$. Pode-se ver a definição de TF na equação 1 .

$$
t f=\frac{\text { frequenciaT }}{N}
$$

O IDF mede quanto um termo é importante. Visto que o TF pode dar importância para termos como "de", "para", "como" por serem termos bastante comuns, aparecem muitas vezes, porém possuem pouca importância. O IDF procura dar um peso maior para termos que ocorrem mais raramente. Pode ser representado pela equação 2 , onde ' $N$ ' é a quantidade total de documentos, e 'n' é a quantidade de documentos que possui um determinado termo.

$$
i d f=\log \left(\frac{N}{n}\right)
$$

Logo uma medida razoável da importância de um termo pode ser obtida utilizando o produto da frequência do termo (TF) com a frequência inversa do documento (IDF) [Salton e Buckley 1988]. A fórmula obtida é representada por:

$$
t f i d f=t f \cdot i d f
$$

\subsection{Pré-processamento}

O pré-processamento é a etapa que é iniciada após a criação ou aquisição da base de dados, é responsável por tratar os dados, de forma que possam ser melhor trabalhados, e para que as técnicas computacionais possam ser aplicadas.

Para a etapa de pré-processamento deste trabalho, três técnicas foram empregadas: tokenização, remoção de stopwords e stemming [Hotho et al. 2005]. A tokenização é um processo separa as palavras de um documento, removendo as pontuações, e substituindo os elementos não textuais, como quebra de linha e tabulação, por um espaço em branco simples.

As stopwords são as palavras que pouco acrescentam à representatividade da coleção de dados, ou que sozinhas nada significam. Exemplos de stopwords são palavras como artigos, pronomes e advérbios. É comum também remover pontuação e caracteres especiais. O conjunto de stopwords é chamado de stoplist. Essa eliminação de stopwords reduz significativamente a 
quantidade de termos, diminuindo o custo computacional das próximas etapas [Rezende et al. 2011]. A Figura 1 mostra um exemplo da aplicação desta técnica.

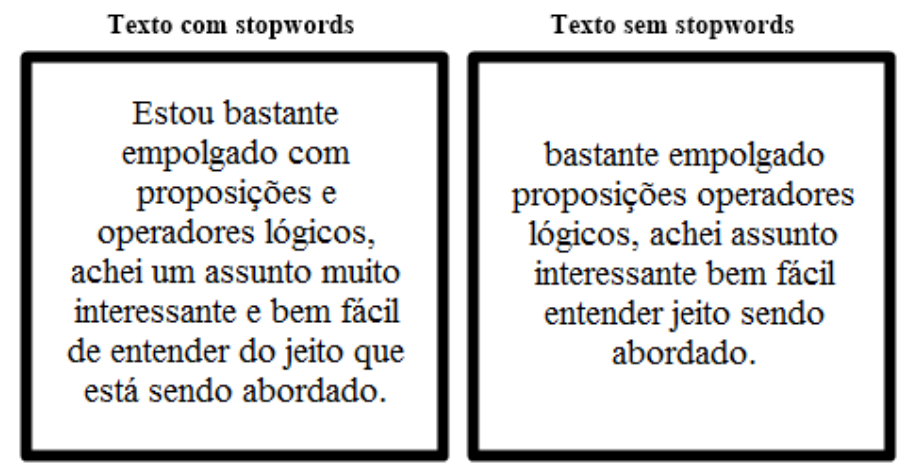

Figura 1 - Exemplo de remoção de stopwords.

O stemming [Hotho et al. 2005] é uma técnica que reescreve as palavras do texto na sua forma básica. A palavra stemming deriva da palavra inglesa stem, que significa basicamente radical, ou seja, esta técnica transforma a palavra na sua forma radical, removendo sufixos. Pode-se observar um exemplo da aplicação da técnica stemming na Figura 2.
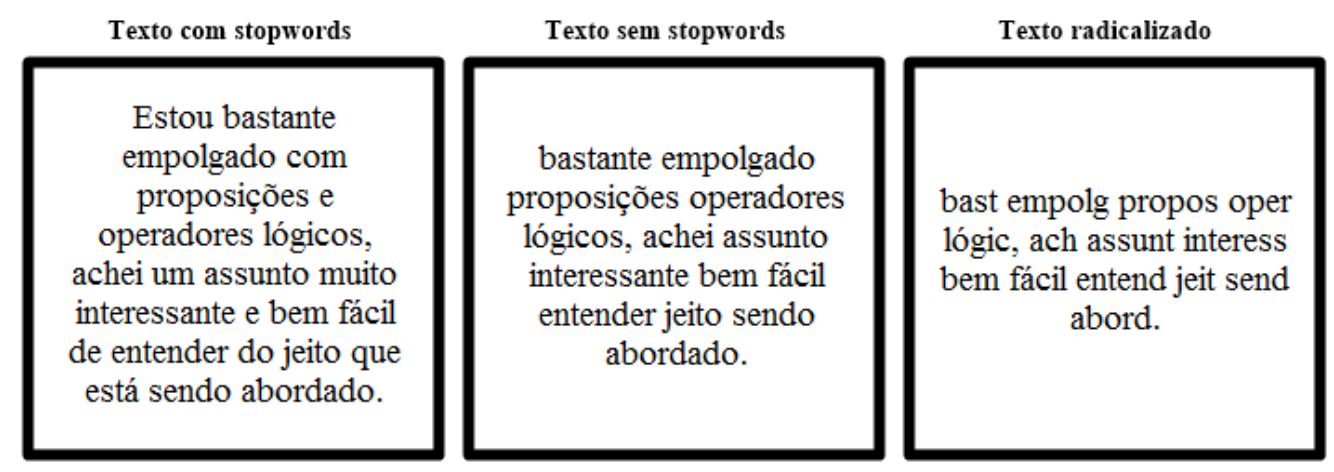

Figura 2 - Exemplo da técnica stemming.

Além destas técnicas, também foi realizada a remoção de termos que tenham uma baixa frequência absoluta em relação à base de dados. Essa remoção é feita porque os termos com baixa frequência normalmente têm pouca influência na identificação das classes definidas. Foram utilizados diferentes limiares para definir o que seria um termo com baixa frequência.

\section{Trabalhos relacionados}

O uso pedagógico de fórum de discussão tem sido viabilizado em diversos trabalhos na literatura de Informática na Educação. A seguir, discutem-se trabalhos similares à abordagem aqui proposta, considerando-se desde propostas mais específicas, cujo foco se aproxima mais do presente trabalho, àquelas mais gerais na tarefa de processamento de fórum.

No trabalho proposto por Ravi e Kim (2007), apresenta-se uma forma de identificar as interações dos alunos nos fóruns educacionais. Ele classifica individualmente as postagens dos alunos como "atos de discurso", que podem ser: complemento (complemento de uma mensagem anterior), informação (informação, comando ou anúncio), correção (correção ou objeção a uma mensagem anterior), elaboração (elaboração de uma mensagem anterior ou descrição, incluindo elaboração de perguntas e respostas), pergunta (uma questão sobre um problema, incluindo questão sobre uma mensagem anterior), resposta (resposta a uma questão anterior, ou sugestão). Para a categorização são utilizadas técnicas de análise, N-grams e SVM (Support Vector 
Machine) para encontrar postagens que sejam categorizadas como pergunta ou resposta, e assim poder auxiliar o professor em encontrar perguntas sem respostas, proporcionando uma gestão mais eficaz das postagens e dúvidas dos alunos.

Lin et al. (2009) propõe um sistema de classificação de gênero das postagens dos fóruns de discussão em ambientes educacionais, utilizando a frequência das palavras como características para o sistema, e aplicando árvore de decisão para classificação. Os gêneros que este sistema tenta identificar são: anúncios (esclarece dúvidas dos outros), perguntas (dada uma informação desconhecida, propõe uma questão), interpretação (interpretação baseada nos fatos e ideias expostas, fazendo predições, análises e reflexões), conflito (opinião conflitante), afirmação (mantendo e defendendo a ideia discordada por outros), e outros (mensagens variadas que são difíceis de categorizar).

Oliveira Júnior et. al. (2011) apresentam uma ferramenta de classificação automática de postagens em fóruns educacionais. As postagens podem ser classificadas como positivas ou negativas, utilizando algoritmos de aprendizado de máquina, como Naive Bayes e SVM (Support Vector Machine). O algoritmo SVM apresenta resultados na avaliação, utilizando as métricas: recall, precision, $F$-measure, porcentagem de mensagens classificadas corretamente e incorretamente, estatística Kappa, erro absoluto médio e raiz do erro quadrático médio. Essa classificação das postagens como positivas (expressam respostas, comentários pertinentes entre outros) e negativas (expressam dúvidas, conteúdo indevido entre outros) auxiliam o professor no momento de fornecer uma atenção maior a um determinado assunto.

Gerosa et. al. (2003) apresentam o AulaNet, um ambiente de interação entre alunos e professores, e demonstra o encadeamento e a organização das postagens através de hierarquia. Essa hierarquia das postagens é derivada da categorização de cada uma delas. As categorias podem ser: seminário (para uma mensagem raiz de uma discussão), questão (para propor um tópico para discussão), argumentação (para responder às questões, fornecendo um ponto de vista), contra argumentação (para oferecer uma posição contrária a uma argumentação), esclarecimento (para solicitar ou esclarecer dúvidas sobre uma mensagem). Esta categorização, implementada pelo próprio AulaNet, é feita manualmente pelo autor da postagem e ajuda a observar os relacionamentos entre as postagens, dando assim subsídios aos professores para que possam coordenar eficazmente essas discussões, e avaliar o desenvolvimento da turma.

Além da abordagem de classificação de postagens, outros sistemas propõem soluções para auxiliar o professor na resolução de dúvidas aplicando diferentes métodos, como:

- A utilização de medidas de similaridade para relacionar dúvidas anteriores com novas perguntas [Heiner e Zachary 2009]. Essa abordagem consegue relacionar inclusive perguntas com diferentes palavras, mas mesmo significado.

- Extração automática de mapas conceituais para análise das postagens de fóruns educacionais [Kowata et al. 2012].

- Utilização de técnicas de mineração de texto baseadas em grafos para extrair informações dos fóruns [Azevedo et al. 2011b].

Apesar de já existirem diferentes soluções na literatura, o sistema proposto possui características que o diferencia dos demais:

- A proposta foi analisada para postagens em português, mas como se trata de uma abordagem estatística pode ser replicada para outros idiomas. A maioria dos trabalhos citados são direcionados para um idioma. 
- Diferentemente dos trabalhos anteriores, foram avaliados diferentes algoritmos e conjuntos de características para encontrar a melhor combinação.

- Além da classificação das postagens é realizada a extração do assunto e recomendação de material.

\section{Abordagem Proposta}

A abordagem proposta no presente trabalho objetiva auxiliar o professor no acompanhamento de postagens de fóruns educacionais. Além disto, o sistema recomenda material auxiliar para estudantes. Para tanto, ela foi dividida em três etapas (Figura 3):

- Classificador automático de postagens, utilizando aprendizagem de máquina. Todas as postagens serão classificadas como dúvida, resposta ou comentário neutro;

- Extrator de assunto de postagens de fóruns educacionais, usando cálculo dos pesos de cada postagem;

- Sistema de recomendação que usa a resposta fornecida pelo extrator, recomendando vídeos do Youtube ${ }^{1}$.

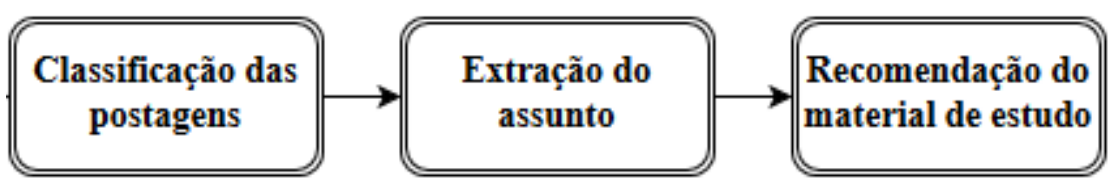

Figura 3 - Fluxo completo do desenvolvimento.

\subsection{Classificação das postagens}

Esta primeira etapa do sistema é um classificador automático de postagens dos fóruns educacionais. As postagens podem ser classificadas como:

- Dúvida: postagem que contenha algum tipo de dúvida sobre o assunto do fórum;

- Neutra: postagem que não acrescenta a discussão, podendo ser apenas um comentário;

- Resposta: postagem que responde a algum questionamento, sendo ele proposto pelo professor, ou uma dúvida expressa por outro aluno.

O fluxo de atividades do classificador é apresentado na Figura 4.

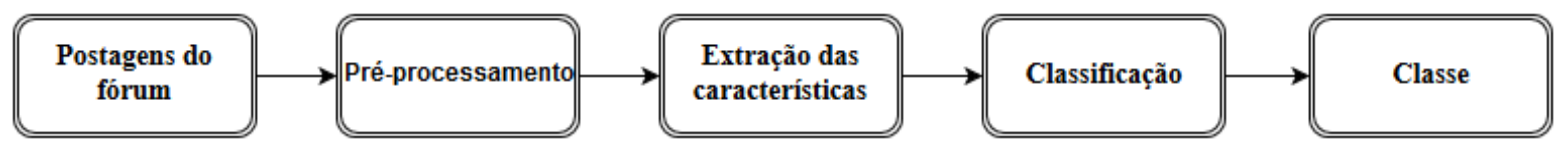

Figura 4 - Fluxo da etapa de classificação das postagens.

\subsubsection{Pré-processamento}

Como técnica de pré-processamento foram aplicadas a tokenização, remoção de stopwords e stemming [Hotho et al. 2005] utilizando a biblioteca $\mathrm{NLTK}^{2}$. A remoção de stopwords eliminará

\footnotetext{
${ }^{1} \mathrm{https}: / /$ www.youtube.com/

2 http://www.nltk.org/
} 
palavras com pouco valor no texto, que porventura podem possuir um TF (term frequency) alto, porém pouca importância no contexto geral. Por outro lado, o stemming colocará as palavras no seu radical, fazendo com que o TF de palavras realmente importantes aumente.

Foram utilizadas diferentes configurações de pré-processamento, utilizando ou não as técnicas de remoção de stopwords e stemming. Em outras palavras, em alguns testes utilizou-se apenas uma técnica ou até mesmo nenhuma das duas. Essas variações foram necessárias para realizar comparações e analisar qual o melhor caso para este projeto, essas comparações são mostradas na Seção 5.3.

\subsubsection{Extração das características}

Após o pré-processamento, é realizada a extração das características que serão os atributos para as técnicas de classificação. Foram definidos dois conjuntos de características. Entre as características utilizadas do primeiro conjunto estão:

- frequência das palavras da classe Dúvida;

- frequência das palavras da classe Neutra;

- frequência das palavras da classe Resposta;

- número de interrogações.

A extração dessas características é feita contando quantas palavras de cada classe aparecem no texto, para cada postagem.

Assim como no pré-processamento, foram feitos testes usando conjuntos de características diferentes, sempre em busca do melhor resultado. As características utilizadas do segundo conjunto são:

- $\quad$ somatório do TF-IDF das palavras pertencentes a classe Dúvida;

- $\quad$ somatório do TF-IDF das palavras pertencentes a classe Neutra;

- somatório do TF-IDF das palavras pertencentes a classe Resposta;

- número de interrogações.

O TF-IDF de cada palavra é calculado e multiplicado pelo número de aparições da palavra, antes do somatório. Uma vez obtidos esses atributos, eles são disponibilizados como entrada para as técnicas de classificação.

A criação desse segundo conjunto é necessária, para que todos os cenários possíveis possam ser explorados. A escolha do TF-IDF nesse segundo conjunto, se deve a sua validação na literatura e por ser uma métrica de peso dos termos bastante utilizada na classificação de texto [Thorsten 1996, Zhang et al. 2011, Jing et al. 2002]

\subsubsection{Classificação}

Para essa etapa foram utilizadas as técnicas de Naive Bayes, indução de árvore de decisão e redes neurais para realizar a classificação das postagens. Todas as técnicas implementadas utilizam uma etapa de treinamento e validação. No processo de avaliação aplicou-se o método de cross-validation (Validação cruzada) [Arlot e Celisse 2010], com o número de folds igual a 10. Esse método divide os dados em vários grupos, dividindo-os entre treinamento e testes.

Foram escolhidas essas técnicas de classificação, pois era preciso técnicas de naturezas diferentes, validadas pela literatura e fossem também comumente utilizadas para classificação textual. 
Para implementação das técnicas foi utilizada a ferramenta Weka (Waikato Environment for Knowledge Analysis) [Hall et al. 2009][Frank et al. 2010], versão 3.6.13. O Weka é uma ferramenta que disponibiliza uma coleção de algoritmos de aprendizado de máquina para utilização na mineração de dados. Nele estão implementadas as técnicas utilizadas no trabalho, assim como outras técnicas de aprendizagem de máquina.

\subsection{Extração do assunto}

Esta é a segunda etapa do sistema proposto, ela é responsável por identificar qual o assunto da dúvida contida na postagem do aluno. Logo após a classificação, sendo a postagem classificada como dúvida, ela entra na etapa de extração. O fluxo dessa etapa é apresentado na Figura 5.

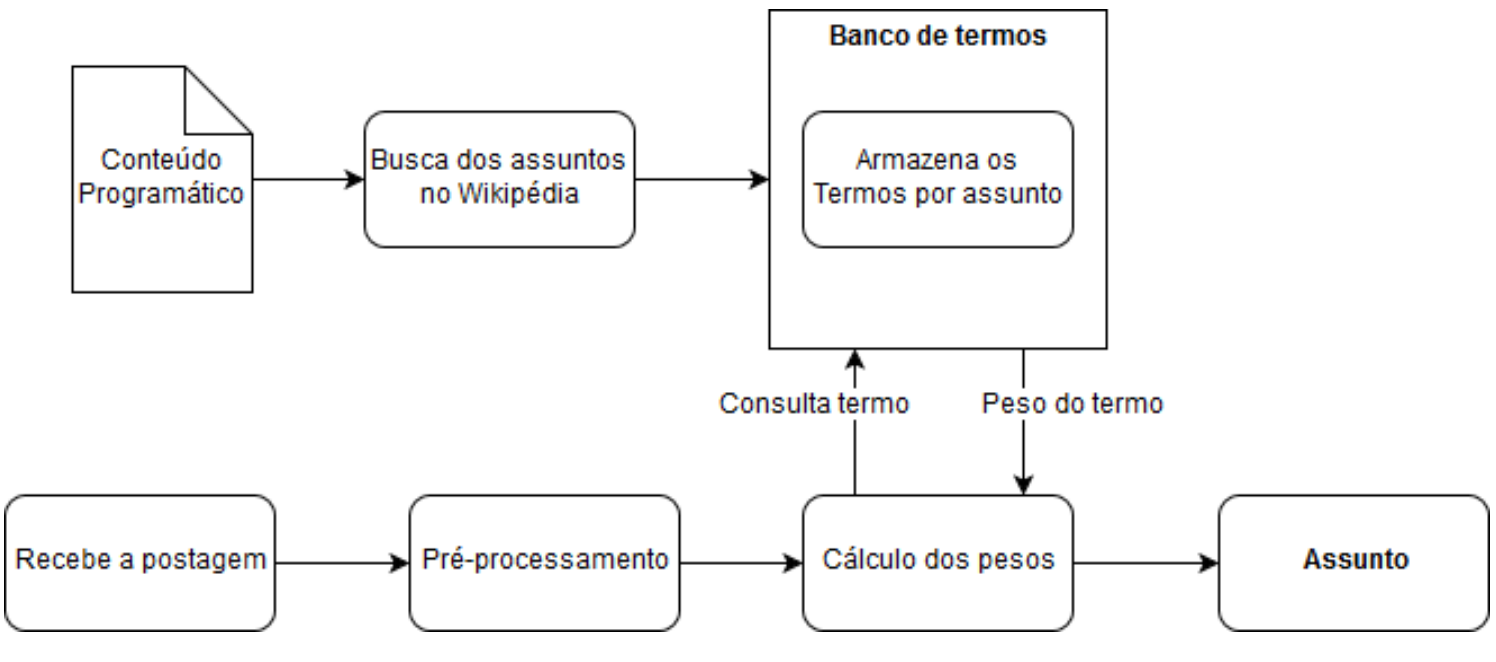

Figura 5 - Fluxo da etapa de extração de assunto.

Esta etapa é dividida em duas partes, a primeira parte recebe como entrada o conteúdo programático de uma disciplina disponibilizado pelo professor. Com isso, todos os assuntos descritos no conteúdo programático são buscados no Wikipédia, e um banco de palavras é criado para cada um dos assuntos, cada palavra possui um peso associado.

A postagem então é comparada com os bancos de palavras, e verifica-se qual tem maior valor pelo somatório dos pesos das palavras. $\mathrm{O}$ assunto em que o somatório dos pesos for maior, é definido como assunto da postagem.

Quando a disciplina é cadastrada no AVA, é necessário também que seja adicionado o plano de trabalho da disciplina, que aborda várias informações sobre ela. Dentre essas informações, existe o conteúdo programático, nele estão todos os assuntos que serão vistos no decorrer da disciplina.

Inicialmente o conteúdo programático é extraído do plano de trabalho da disciplina de forma automática. Em seguida os assuntos são organizados hierarquicamente, como assuntos 'pai' e assuntos 'filho', como os próprios nomes sugerem, os assuntos indicados como 'filho' são derivações do assunto 'pai'. A Figura 6 mostra um exemplo de hierarquia dos assuntos. 


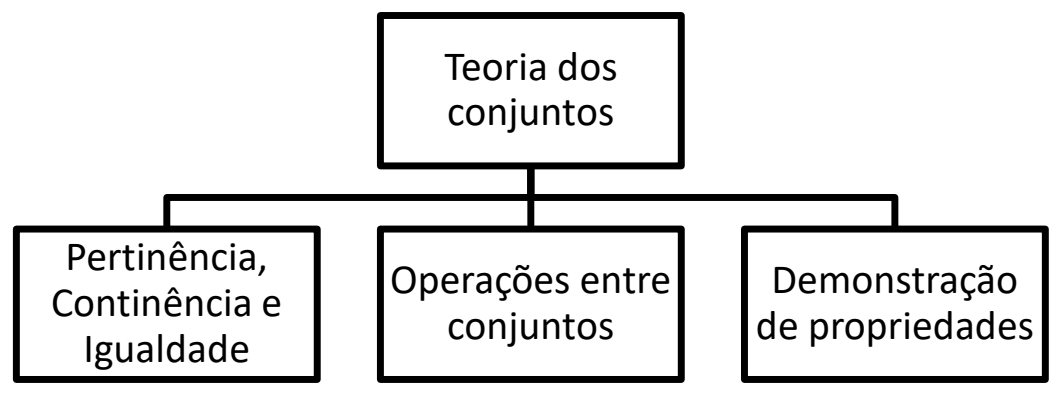

Figura 6 - Exemplo da hierarquia dos assuntos.

Como pode-se observar na Figura 6, o assunto "Teoria dos conjuntos" possui um nível hierárquico maior (assunto "pai”), e os outros no nível abaixo, são os assuntos "fillho".

Para cada assunto, seja ele 'pai' ou 'filho', é feita uma consulta no Wikipédia de cada um deles. Todos os hiperlinks e as palavras em negrito são adicionados no banco de termos de seu respectivo assunto, juntamente com seu peso. O peso é calculado pela equação do TF (e.g. equação 1), que é razão entre a quantidade de aparições do termo e a quantidade total de termos no texto do Wikipédia.

Como na etapa de classificação, foram utilizadas as técnicas de remoção de stopwords e o stemming. Cada postagem é submetida à ambas as técnicas, assim como os termos adicionados no banco de termos de cada assunto.

Assim como explicado anteriormente, os pesos dos termos correspondentes a cada assunto, é a razão entre a quantidade de aparições do termo e a quantidade total de termos no texto do Wikipédia.

Com todos os bancos de termos preenchidos, e cada termo com seu respectivo peso, é possível então definir o assunto de uma postagem. Para isso cada palavra contida na postagem é comparada com os bancos. Para cada termo da postagem que esteja contido no banco de termos, o peso do termo do banco é somado. Ao termino de cada banco o somatório é armazenado e zerado, para que o próximo somatório possa iniciar. O somatório do banco de termos que possuir maior valor, é o assunto da postagem.

\subsection{Recomendação de material de estudo}

Esta é a última etapa do sistema, nesta etapa, é dado como entrada o assunto extraído da postagem assim como visto na etapa anterior, e é recomendado um vídeo do Youtube que melhor atenda o assunto.

Após a etapa de extração do assunto da postagem, o assunto é passado como argumento para a função responsável por buscar vídeos no Youtube. Essa função retorna o link do vídeo melhor ranqueado, o algoritmo do Youtube por padrão ordena a lista de vídeos por relevância, logo, é suposto que seja o melhor para a pesquisa realizada. Ao obter este link, ele é recomendado ao aluno, servindo assim como auxílio para que o aluno possa sanar sua dúvida.

\section{Metodologia e Resultados Experimentais}

Esta seção apresenta a metodologia seguida para avaliar a abordagem proposta, ressaltando as bases de dados utilizadas no experimento, as métricas de avaliação, assim como se discutem os resultados obtidos nos testes realizados nas três etapas: classificação da postagem, extração do assunto da postagem, e recomendação de material de estudo. 


\subsection{Bases de dados}

Esta etapa do trabalho diz respeito a criação da base de dados utilizadas neste projeto. Durante o processo de desenvolvimento desta fase, de classificação, do projeto foram utilizadas 3 bases de dados diferentes.

A base de dados 1 foi retirada do AVA da UFAL (Universidade Federal de Alagoas), e as postagens foram retiradas de várias disciplinas diferentes do curso à distância de bacharelado em sistemas de informação. Já a base de dados 2, foi retirada também do AVA da UFAL, porém, das disciplinas de algoritmos e estruturas de dados I e II, do mesmo curso. A base de dados 2 é a única que será utilizada tanto na etapa de classificação da postagem quanto na etapa de extração de assunto, por possuir anotação das classes e por ser composta de postagens de uma única disciplina, sendo assim possível extrair seu assunto.

A Tabela 1 mostra a distribuição das classes nas bases de dados utilizadas em toda fase de classificação do projeto, onde os valores expostos representam as respectivas quantidades de postagens pertencentes às classes indicadas.

Tabela 1 - Composição das bases de dados 1 e 2.

\begin{tabular}{c|cc}
\hline \multirow{2}{*}{ Classes } & \multicolumn{2}{|c}{ Bases de dados } \\
\cline { 2 - 3 } & $B D 1$ & BD2 \\
\hline Dúvidas & 198 & 200 \\
Neutras & 104 & 200 \\
Respostas & 188 & 200 \\
\hline Total & 490 & 600 \\
\hline
\end{tabular}

A terceira base de dados (base de dados 3) possui 387 postagens, e diferentemente das outras duas, não está distribuída conforme as classes Dúvida, Neutra ou Resposta. A distribuição desta base é feita conforme o assunto ao qual pertencem. Pode-se ver a distribuição na Tabela 2.

Tabela 2 - Composição da base de dados 3.

\begin{tabular}{l|c}
\hline \multicolumn{1}{c|}{ Classes } & BD3 \\
\hline Relações, funções e sequências & 122 \\
Lógica & 118 \\
Teoria dos números & 38 \\
Teoria dos conjuntos & 119 \\
\hline \multicolumn{1}{c|}{ Total } & 397 \\
\hline
\end{tabular}

Esta base de dados foi desenvolvida na disciplina de tópicos avançados em inteligência artificial, do curso de bacharelado em ciência da computação da UFRPE (Universidade Federal Rural de Pernambuco). Os alunos dessa disciplina tiveram que escrever postagens que apresentassem dúvidas ou respostas com relação aos assuntos da disciplina de matemática discreta. Essa base foi distribuída segundo o assunto que representavam na disciplina proposta. Os valores apresentados na Tabela 2 representam a quantidade de postagens que os assuntos contêm.

Todas as bases foram categorizadas manualmente por dois avaliadores. Em caso de discordância entre os avaliadores, um terceiro foi consultado para resolver a divergência. 


\subsection{Métricas de avaliação}

Para avaliar os classificadores foi utilizada a métrica de avaliação F-Measure (medida-F) [Friedman et al. 1997]. F-Measure pode ser interpretada como uma média ponderada da precisão e da cobertura, onde a precisão avalia a quantidade de instâncias que foram classificadas corretamente e a cobertura avalia a porcentagem instâncias de uma determinada classe que não foi classificada como pertencente a essa classe.

Outra métrica que foi utilizada foi a acurácia, que é definida como sendo a razão do total de elementos classificados corretamente pelo total de elementos desta classe. Esta métrica foi escolhida para avaliar a extração dos assuntos das postagens.

\subsection{Classificação das postagens}

Para escolher uma técnica de classificação a fim de atingir o objetivo apresentado, investiu-se em estudo comparativo sobre as técnicas: Naive Bayes, Indução de Árvore de Decisão e Redes Neurais, tendo essas sido realizadas, respectivamente, com as seguintes implementações da ferramenta Weka: NaiveBayes, J48 e MultilayerPerceptron. É importante destacar que foram utilizados os parâmetros padrões do Weka para todos os algoritmos.

Para a realização deste primeiro teste, não foi aplicado nenhum tipo de préprocessamento nas postagens. As características selecionadas foram as frequências de palavras de cada classe (Dúvida, Neutra, Resposta), e o limite de corte foi deixado em 0, podendo-se observar o resultado na Tabela 3. A avaliação da classificação foi feita usando-se a métrica Fmeasure. Vale salientar que o $\mathrm{BD} 3$ não foi utilizado nesta comparação por não estar categorizado em pergunta, resposta ou comentário neutro. O objetivo do BD3 é avaliar o assunto da postagem, que é na próxima etapa do sistema.

Tabela 3 - Média das medidas-F das técnicas de classificação

\begin{tabular}{|c|c|c|}
\hline \multirow{2}{*}{ Técnicas de classificação } & \multicolumn{2}{|c|}{ Média da F-measure } \\
\hline & $B D 1$ & $B D 2$ \\
\hline NaiveBayes & 0.526 & 0.701 \\
\hline $\mathrm{J} 48$ & 0.885 & 0.882 \\
\hline MultilayerPerceptron & 0.962 & 0.972 \\
\hline
\end{tabular}

Ao analisar os resultados, chega-se à conclusão que a rede neural MLP é a mais indicada para a classificação de postagens de fóruns educacionais, dentre as três técnicas analisadas, alcançando os valores de 0.962 e 0.972 para BD1 e BD2 respectivamente. A conclusão de ter um bom classificador para o problema impacta na possibilidade de identificar automaticamente o tipo de postagem do fórum, possibilitando ser integrado a um sistema que ajude o aluno quando houver postagem de dúvida. $\mathrm{O}$ resultado confirma a conclusão de Rolim et al. [Rolim et al. 2014], que aponta o MLP como melhor algoritmo classificador para a classificação de postagens.

Com o melhor algoritmo definido, resta definir quais serão as características que devem ser selecionadas, quais técnicas de pré-processamento devem ser empregadas, e qual limite de corte deve ser estabelecido, a fim de que possa ser encontrado o melhor cenário possível.

Como o principal objetivo do sistema é encontrar dúvidas, o melhor resultado, será o que possuir o maior valor da F-measure da classe Dúvida. 
Ao utilizar como características as frequências das palavras foi definido 3 cenários diferentes, são eles:

- Cenário 1: Classificação sem técnicas de pré-processamento;

- Cenário 2: Classificação usando remoção de stopwords;

- Cenário 3: Classificação usando remoção de stopwords e stemming.

Os limites de cortes utilizados foram definidos com base nos limites utilizados em Rolim et al. [Rolim et al. 2014] e variaram entre '0', '5' e '10'. Pode-se observar os valores da Fmeasure alcançados para cada classe de acordo com a base de dados utilizadas na Tabela 4.

Tabela 4 - F-measure das classes de acordo com a base de dados (Frequência).

\begin{tabular}{|c|c|c|c|c|c|c|c|c|c|c|}
\cline { 3 - 11 } \multicolumn{2}{c|}{} & \multicolumn{3}{c|}{ Limite de corte 0 } & \multicolumn{3}{c|}{ Limite de corte 5 } & \multicolumn{3}{c|}{ Limite de corte 10 } \\
\cline { 3 - 11 } \multicolumn{2}{c|}{} & Dúvida & Neutra & Reposta & Dúvida & Neutra & Reposta & Dúvida & Neutra & Reposta \\
\hline \multirow{3}{*}{ BD1 1 } & Cenário 1 & $\mathbf{0 , 9 6}$ & 0,935 & 0,979 & 0,738 & 0,708 & 0,774 & 0,728 & 0,623 & 0,745 \\
\cline { 2 - 11 } & Cenário 2 & $\mathbf{0 , 9 6 3}$ & 0,953 & 0,966 & 0,793 & 0,768 & 0,801 & 0,736 & 0,634 & 0,71 \\
\cline { 2 - 11 } & Cenário 3 & $\mathbf{0 , 8 9 2}$ & 0,825 & 0,922 & 0,779 & 0,777 & 0,806 & 0,77 & 0,713 & 0,782 \\
\hline \multirow{3}{*}{ BD2 2} & Cenário 1 & $\mathbf{0 , 9 7 7}$ & 0,961 & 0,977 & 0,915 & 0,844 & 0,835 & 0,821 & 0,797 & 0,827 \\
\cline { 2 - 10 } & Cenário 2 & $\mathbf{0 , 9 6 2}$ & 0,933 & 0,955 & 0,906 & 0,859 & 0,872 & 0,891 & 0,823 & 0,834 \\
\cline { 2 - 10 } & Cenário 3 & 0,872 & 0,874 & 0,895 & $\mathbf{0 , 8 7 9}$ & 0,809 & 0,854 & 0,842 & 0,785 & 0,796 \\
\hline
\end{tabular}

O cenário 1 e o cenário 2 apresentaram os maiores valores da F-measure da classe dúvida tanto na BD1 quanto na BD2 quando o limite de corte estava em 0.

Assim foram definidos 3 cenários diferentes, porém utilizando o TF-IDF como características, são eles:

- Cenário 4: Classificação sem técnicas de pré-processamento;

- Cenário 5: Classificação usando remoção de stopwords;

- Cenário 6: Classificação usando remoção de stopwords e stemming.

O limite de corte, variou entre os valores ' 0 ', ' 0,00109 ' e ' 0,00247 '. Os limites de cortes utilizados foram definidos de forma empírica após o teste de vários outros valores, sendo que esses foram os que apresentaram os melhores resultados. A Tabela 5 mostra os valores da Fmeasure para cada classe nas duas bases de dados.

Tabela 5 - F-measure das classes de acordo com a base de dados (TF-IDF).

\begin{tabular}{|c|c|c|c|c|c|c|c|c|c|c|}
\cline { 3 - 11 } \multicolumn{2}{c|}{} & \multicolumn{3}{c|}{ Limite de corte 0 } & \multicolumn{3}{c|}{ Limite de corte 0,00109 } & \multicolumn{2}{c|}{ Limite de corte 0,00247 } \\
\cline { 3 - 11 } \multicolumn{2}{c|}{} & Dúvida & Neutra & Reposta & Dúvida & Neutra & Reposta & Dúvida & Neutra & Reposta \\
\hline \multirow{3}{*}{ BD11 1} & Cenário 4 & 0,832 & 0,48 & 0,636 & $\mathbf{0 , 8 5 4}$ & 0,819 & 0,795 & 0,834 & 0,705 & 0,748 \\
\cline { 2 - 11 } & Cenário 5 & 0,832 & 0,879 & 0,78 & $\mathbf{0 , 9 1 1}$ & 0,898 & 0,87 & 0,855 & 0,763 & 0,788 \\
\cline { 2 - 11 } & Cenário 6 & 0,823 & 0,667 & 0,711 & $\mathbf{0 , 9 0 8}$ & 0,916 & 0,87 & 0,868 & 0,849 & 0,823 \\
\hline \multirow{3}{*}{ BD2 } & Cenário 4 & $\mathbf{0 , 9 3 8}$ & 0,908 & 0,895 & 0,885 & 0,817 & 0,777 & 0,846 & 0,655 & 0,585 \\
\cline { 2 - 11 } & Cenário 5 & 0,85 & 0,549 & 0,536 & $\mathbf{0 , 9 4 1}$ & 0,899 & 0,895 & 0,893 & 0,822 & 0,8 \\
\cline { 2 - 11 } & Cenário 6 & 0.843 & 0.639 & 0.611 & $\mathbf{0 . 9 2 6}$ & 0.885 & 0.88 & 0.882 & 0.793 & 0.801 \\
\hline
\end{tabular}

Ao observar os resultados apresentados, é possível notar que os dois primeiros cenários (usando frequência de palavras) obtiveram os melhores resultados, tanto na BD1 quanto na BD2, com os valores de 0.96 e 0.963 para a BD1, e 0.977 e 0.962 para a BD2. Observa-se nas Tabelas 4 e 5 que os cenários 1 e 2 são os que apresentam os maiores valores da F-measure em ambas as bases de dados. 
O cenário 1 faz uso da frequência das palavras das classes como característica, e não faz uso de técnicas de pré-processamento. Os valores apresentados na Tabela 4 para o cenário 1 , foram alcançados quando o limite de corte estava em 0 .

O cenário 2, faz uso da frequência das palavras das classes, e faz a remoção de stopwords como técnica de pré-processamento. Assim como o cenário 1, os melhores valores foram atingidos quando o limite de corte estava em 0.

Ao calcular a média dos valores atingidos na BD1 e BD2 do cenário 1 e 2, chega-se aos valores de 0.968 e 0.962 respectivamente. $\mathrm{O}$ valor da média do cenário 1 é levemente superior à média do cenário 2. Portanto, o cenário 1 é o mais adequado para ser utilizado na etapa de classificação das postagens.

\subsection{Extração do assunto das postagens}

Como explicado na Seção 5.1, foram utilizadas duas bases de dados (BD2 e BD3) com o intuito de avaliar a acurácia do algoritmo de extração de assunto. Para os testes realizados, foi necessário também o conteúdo programático dos cursos das quais as bases estão relacionadas, no caso, algoritmos e estrutura de dados para a BD2, e matemática discreta para a BD3.

Foi possível extrair corretamente o assunto de $66,66 \%$ das postagens. Contudo, foi identificado que algumas postagens dificultam a extração do seu assunto corretamente, tais como: "Professor, qual seria a melhor forma para resolver a primeira questão da lista?"; "Que horas abre o laboratório?”; "Qual será a data da prova?”.

Esses tipos de postagens dificultam a extração do seu assunto, sabendo que para uma extração correta, precisa-se que o assunto esteja relacionado com algum assunto no conteúdo programático da disciplina. Outro teste foi realizado, removendo esses tipos de postagens de forma manual, e a acurácia subiu para 90,58\%. Este resultado mostra que a eliminação das postagens que não são relacionadas a algum assunto melhora a extração.

O resultado do texto no BD3 pode ser observado na Tabela 6. A média da acurácia obtida foi de 76,1\%. Analisando as partes que compõem este resultado, observa-se que os resultados individuais foram diferentes, um exemplo dessa diferença pode ser visto pela acurácia de "Relações, funções e sequências", 62,3\%, e pela taxa de "Lógica" de 86,44\%.

Tabela 6 - Taxas de acerto dos assuntos da base de dados 1.

\begin{tabular}{l|c}
\multicolumn{1}{c|}{ Assunto } & Acurácia \\
\hline Teoria dos conjuntos & $71,43 \%$ \\
Teoria dos números & $84,21 \%$ \\
Relações, funções e sequências & $62,3 \%$ \\
Lógica & $86,44 \%$ \\
\hline \multicolumn{2}{c}{ Média } \\
\hline
\end{tabular}

Esses resultados nos mostram que mesmo com assuntos do mesmo universo, no caso, matemática discreta, houve uma diferença considerável entre os resultados. Essa diferença pode ser creditada a como o conteúdo programático da disciplina é descrito. 


\section{Conclusão}

Apresentou-se neste artigo uma nova abordagem computacional com ferramentas para ajudar o professor a solucionar dúvidas dos alunos, de forma que pudesse agilizar e facilitar o processo de ensino-aprendizagem. Com essa abordagem, buscou-se contribuir para o estado da arte no contexto de educação online, auxiliando tanto o professor, reduzindo o tempo empregado para responder todos os questionamentos dos alunos, quanto o aluno, indicando materiais de estudo que possam auxiliá-lo na resolução da sua dúvida. Assim, foi proposta a utilização de técnicas de aprendizagem de máquina para identificação automática de dúvidas e extração de conteúdo das postagens. Essas informações foram usadas para recomendação de conteúdo para auxiliar a solução de dúvidas dos alunos nos fóruns educacionais. Essa abordagem busca dar dinamicidade às interações do fórum, e consequentemente aumentar a velocidade da resolução das dúvidas dos alunos e dos problemas propostos pelos professores. Como um dos resultados importantes, alcançou-se uma acurácia na identificação de dúvidas de $97 \%$ e na extração do assunto da dúvida de $76,1 \%$.

Como trabalhos futuros, pretende-se: (i) desenvolver uma forma de eliminar as postagens de dúvidas que não são relacionadas com algum assunto do conteúdo programático da disciplina; (ii) realizar experimentos de extração de assuntos, utilizando outras fontes além do Wikipédia para coletar os termos relacionados; (iii) realizar um estudo sobre a adequação do algoritmo MLP ao problema apresentado neste trabalho.

\section{Referências}

Akyuz, H. I., Kurt, M. (2010). Effect of teacher's coaching in online discussion forums on students' perceived self-efficacy for the educational software development. Procedia Social and Behavioral Sciences, World Conference on Learning, Teaching and Administration Papers, vol. 9, no. 0, pp. 633 - 637. doi: 10.1016/j.sbspro.2010.12.209 [GS $\underline{\text { Search }}$

Arlot, S., Celisse, A., et al. (2010). A survey of cross-validation procedures for model selection. Statistics surveys, 4:40-79. [GS Search]

Azevedo, B. F. T., Behar, P. A., Reategui, E. B. (2011a). Análise temática das mensagens de discussões online, Cadernos de Informática - Volume 6 - Número 1. [GS Search]

Azevedo, B. F. T., Behar, P. A., e Reategui, E. B. (2011b). Análise das mensagens de fóruns de discussão através de um software para mineração de textos. Simpósio Brasileiro de Informática na Educação-SBIE. Vol. 1. No. 1. Available from http://www.brie.org/pub/index.php/sbie/article/view/1572 [GS Search]

Baeza-Yates, R. e Ribeiro-Neto, B. (2013). Recuperação de informação: conceitos e tecnologia das máquinas de busca. Bookman editora, $2^{a}$ edição, 2013. [GS Search]

Baker, R. e Yacef, K. (2009). The state of educational data mining in 2009: A review and future visions. Journal of Educational Data Mining, vol. 1, no. 1, pp. 3-17. Available from https://jedm.educationaldatamining.org/index.php/JEDM/article/view/8 [GS Search]

Barros, M. G. e Carvalho, A. B. G. (2011). As concepções de interatividade nos ambientes virtuais de aprendizagem. Tecnologias digitais na educação, 1(1):209-232. [GS Search]

Batista, E. M., \& Gobara, S. T. (2011). O fórum on-line e a interação em um curso a distância. RENOTE, 5(1). [GS Search] 
Cheng, C. K., Par, D. E., Collimore, L.-M., and Joordens, S. (2011). Assessing the effectiveness of a voluntary online discussion forum on improving students course performance. Computers \& Education, 56(1):253 - 261. doi: 10.1016/j.compedu.2010.07.024 [GS Search]

De Wever, B., Schellens, T., Valcke, M., e Van Keer, H. (2006). Content analysis schemes to analyze transcripts of online asynchronous discussion groups: A review. Computers \& Education, 46(1), 6-28. doi: 10.1016/j.compedu.2005.04.005 [GS Search]

Frank, E., Hall, M., Holmes, G., Kirkby, R., Pfahringer, B., Witten, I. H., e Trigg, L. (2010). Weka-a machine learning workbench for data mining. Data Mining and Knowledge Discovery Handbook, pp. 1269-1277. [GS Search]

Freitas, M. A. S. (2009). Avaliação da Aprendizagem em ambientes de formação online: aportes para uma abordagem hermenêutica. PhD thesis, UFBA: Faculdade de Educação. [GS $\underline{\text { Search }}$

Friedman, N., Geiger, D., e Goldszmidt, M. (1997). Bayesian network classifiers. Machine learning, 29(2-3):131-163. doi: 10.1023/A:1007465528199 [GS Search]

Gerosa, M. A., Pimentel, M. G., Fucks, H. e Lucena, C. J. P. (2003). Coordenação de Fóruns Educacionais: Encadeamento e Categorização de Mensagens. XIV Simpósio Brasileiro de Informática na Educação. Available from ie.org/pub/index.php/sbie/article/view/234 [GS Search]

Hall, M., Frank, E., Holmes, G., Pfahringer B., Reutemann P., e Witten I. H. (2009). The weka data mining software: an update, ACM SIGKDD explorations newsletter, vol. 11, no. 1, pp. 10-18. doi: $10.1145 / 1656274.1656278$ [GS Search]

Haykin S. (1999). Neural Networks: A Comprehensive Foundation. 2a Edição. Macmillan, Nova Iorque. [GS Search]

Heiner, C., e Zachary, J. (2009). Improving Student Question Classification. In Educational Data Mining 2009. [GS Search]

Hotho, A., Nurnberger, A., e Paas, G. (2005). A brief survey of text mining. LDV Forum GLDV Journal for Computational Linguistics and Language Technology, 20(1):19-62. Available from http://www.jlcl.org/2005_Heft1/19-62_HothoNuernbergerPaass.pdf [GS $\underline{\text { Search] }}$

Jain, P. "Virtual learning environment." International Journal in IT \& Engineering 3.5 (2015): 75-84. [GS Search]

Jing, L-P., Huang, H-K., Shi, H-B. (2002). Improved feature selection approach TFIDF in text mining. In: Machine Learning and Cybernetics. Proceedings. 2002 International Conference on. IEEE, 2002. p. 944-946. doi: 10.1109/ICMLC.2002.1174522 [GS Search]

Kowata, J. H., Cury, D., e Boeres, M. C. S. (2012). Em direção à construção automática de Mapas Conceituais a partir de textos. Revista Brasileira de Informática na Educação, 20(1), 33. doi:10.5753/rbie.2012.20.1.33 Available from $\underline{\mathrm{http}: / / \mathrm{br}-}$ ie.org/pub/index.php/rbie/article/view/1367 [GS Search]

Lin, F.-R., Hsieh, L.-S., Chuang, F.-T. (2009). Discovering genres of online discussion threads via text mining. Computers \& Education, vol. 52, no. 2, pp. 481-495. : $\underline{10.1016 / \text { j.compedu.2008.10.005 [GS Search] }}$

Mazzolini, M., e Maddison, S. (2007). When to jump in: The role of the instructor in online discussion forums. Computers \& Education, 49(2), 193-213. doi: 10.1016/j.compedu.2005.06.011 [GS Search] 
Mohamad, S. K. e Tasir, Z. (2013). Educational data mining: A review. Procedia - Social and Behavioral Sciences, The 9th International Conference on Cognitive Science, vol. 97, no. 0, pp. 320 - 324. [GS Search]

Oliveira Júnior, R. L., Esmin, A. A., Coelho, T. A., Araújo, D. L, Silva, L. A., Giroto, R. (2011). Uma Ferramenta de Monitoramento Automático de Mensagens de Fóruns em Ambientes Virtuais de Aprendizagem. Anais do XXII SBIE - XV WIIE. [GS Search]

Pena-Shaff, J. B., \& Nicholls, C. (2004). Analyzing student interactions and meaning construction in computer bulletin board discussions. Computers \& Education, 42(3), 243265. doi:10.1016/j.compedu.2003.08.003 [GS Search]

Quinlan, J. R. (2014). C4. 5: programs for machine learning. Elsevier. [GS Search]

Ravi, S., \& Kim, J. (2007). Profiling student interactions in threaded discussions with speech act classifiers. Frontiers in Artificial Intelligence and Applications, 158, 357. [GS Search]

Rezende, S. O., Marcacini, R. M., e Moura, M. F. (2011). "O uso da mineração de textos para extração e organização não supervisionada de conhecimento". Revista de Sistemas de Informação da FSMA, no. 7, pp. 7-21. [GS Search]

Rigo, S. J., Flores, E. M., Abech, M., Barbosa, J. L., Costa, C., Martins, L. L., e Araujo, D. A. (2013). O papel do Processamento de Língua Natural e da Representação de Conhecimento na extração de informações em mensagens textuais na Educação a Distância?. Simpósio Brasileiro de Informática na Educação-SBIE. Vol. 24, No. 1, p. 647. doi: 10.5753/cbie.sbie.2013.647 [GS Search]

Rolim, V., Cordeiro, F. R., Ferreira, R. (2014). Reconhecimento de Padrões Aplicados a Comentários de Fóruns Educacionais. Encontro nacional da inteligência artificial e computacional. Available from http://www.lbd.dcc.ufmg.br/colecoes/eniac/2014/003.pdf [GS Search]

Rolim, V., Ferreira, R. e Costa, E. (2016a). Identificação Automática de Dúvidas em Fóruns Educacionais. Simpósio Brasileiro de Informática na Educação-SBIE. Vol. 27. No. 1. doi: 10.5753/cbie.sbie.2016.936 [GS Search]

Rolim, V., Ferreira, R., e Costa, E. (2016b). Método Supervisionado para Identificação de Dúvidas em Fóruns Educacionais. Anais dos Workshops do Congresso Brasileiro de Informática na Educação. Vol. 5. No. 1. doi:10.5753/cbie.wcbie.2016.102 [GS Search]

Romero, C., López, M. I., Luna, J. M., e Ventura, S. (2013). Predicting students' final performance from participation in on-line discussion forums. Computers \& Education, 68, 458-472. doi:10.1016/j.compedu.2013.06.009 [GS Search]

Rutten, A. L. B. (2010). Bayes' theorem: scientific assessment of experience. International jornal of high dilution research, vol. 9, no. 32. [GS Search]

Salton, G. e Buckley, C. (1988). Term-weighting approaches in automatic text retrieval. Journal information processing and management: an international Journal, vol. 24, cap. 5, pp. 513523. doi:10.1016/0306-4573(88)90021-0 [GS Search]

Sebastiani, F. (2002). Machine learning in automated text categorization. ACM Computing Surveys, Vol. 34, No. 1, pp. 1-47. doi:10.1145/505282.505283 [GS Search]

Thorsten, J. (1996). A Probabilistic Analysis of the Rocchio Algorithm with TFIDF for Text Categorization. Carnegie-mellon univ pittsburgh pa dept of computer science. [GS Search]

Yoo, J., e Kim, J. (2014). Can online discussion participation predict group project performance? investigating the roles of linguistic features and participation patterns. 
International Journal of Artificial Intelligence in Education, 24(1), 8-32. doi: $\underline{10.1007 / \mathrm{s} 40593-013-0010-8}$ [GS Search]

Weiss, S. M. e Kulikowski, C. A. (1991). Computer Systems that Learn: Classification and Prediction Methods from Statistics, Neural Nets, Machine Learning, and Expert Systems. Morgan Kaufmann Publishers Inc., São Francisco, CA, USA. [GS Search]

Zhang, W., Yoshida, T., Tang, X. (2011). A comparative study of TF* IDF, LSI and multiwords for text classification. Expert Systems with Applications, v. 38, n. 3, p. 2758-2765. doi:10.1016/i.eswa.2010.08.066 [GS Search] 